\title{
Modelling studies of charged particle interactions for a space application
}

\author{
Karen L. Aplin and Vladimir P. Tarakanov* \\ Space Science and Technology Department, Rutherford Appleton Laboratory, \\ Chilton, Didcot, Oxfordshire OX11 0QX \\ * High Energy Research Centre, Moscow, Russia
}

\begin{abstract}
An electron emitter neutraliser to prevent spacecraft charging from the use of positive ion thrusters is under development at the Rutherford Appleton Laboratory. A particle-in-cell code, KARAT, has been used to simulate laboratory experiments. Laboratory test results have been successfully obtained with simulations, for model verification. Neutraliser-ion thruster interactions in space have also been modelled. An ion-propelled spacecraft rapidly charges to kilovolt potentials, which can be dissipated by an electronemitting neutraliser. The effect of background particle concentrations on neutralisation has also been studied.
\end{abstract}

\section{Introduction}

Application of new satellite technology for fundamental physics experiments in space requires precise adjustment of the satellite's position, making micropropulsion an important new challenge in spacecraft engineering. One propulsion method is to accelerate heavy ions such as caesium (Ce), away from the spacecraft by a high electric field, a technique known as Field Emission Electric Propulsion (FEEP). A consequence of FEEP is that the loss of ions from the spacecraft results in a large static charge, reducing propulsion efficiency. The Rutherford Appleton Laboratory (RAL) has been contracted by the European Space Agency (ESA) to produce an engineering model of a FEEP neutraliser. A concurrent ESA contract with a different laboratory is funding development of a FEEP thruster, which precludes prototype and breadboard testing of the ion thruster and neutraliser together until both have been constructed as engineering models. Modelling studies are required to assess issues arising from electron-ion interactions for neutraliser design, and are also of use to simulate ion thrusters and neutralisers in space, where direct measurements are difficult.

The KARAT particle-in-cell (PIC) code is ideal for such applications. It solves Maxwell's equations and equations of motion for axisymmetric 2-D geometries. KARAT has proved successful in microwave work, and has been used to simulate astrophysical plasmas [1] and atomic cluster-plasma interactions [2]. In this paper KARAT is used to model the laboratory test set-up, to increase both understanding of the test results and confidence in the model output for the space application. Neutraliser function in the space environment has also been simulated.

\section{The KARAT model}

KARAT is an electromagnetic PIC code. In this paper the 2-D version for an axisymmetric system (including three electromagnetic field and momentum components) has been used. The code also includes inelastic processes for particle-gas reactions. Geometry and particle characteristics such as mass, energy and charge are defined by the user. The model assumes that all objects have homogeneous conductivity [3]. For this application, a $\mathrm{Ce}^{+}$thruster 
emitting $10 \mathrm{keV}$ ions has been assumed throughout. Particles are emitted from pre-defined parts of the spacecraft surface and have a Dirac-delta energy distribution (all particles have the same energy). Particles are shown on the figures as dots representing the PIC-particles used in model calculations. The ratio between the number of model particles and displayed particles is defined by a merging factor, $M$; when $M=1$ the ratio is $3 \times 10^{9}$ ( $M$ is typically 0.001 in this paper).

\section{Spacecraft charging by an ion thruster}

A simple model of a spacecraft propelled by a FEEP thruster was set up to observe the electrical effects of the thruster. The spacecraft is modelled as cylindrical with a radius of $2.5 \mathrm{~m}$ and a depth of $2 \mathrm{~m}$. The boundaries are much greater than the satellite dimensions and are set at ground potential (as is the spacecraft) at the start of the simulation, Figure 1. The thruster is assumed to be a uniform $\mathrm{Ce}^{+}$beam, as described in section 1 above.

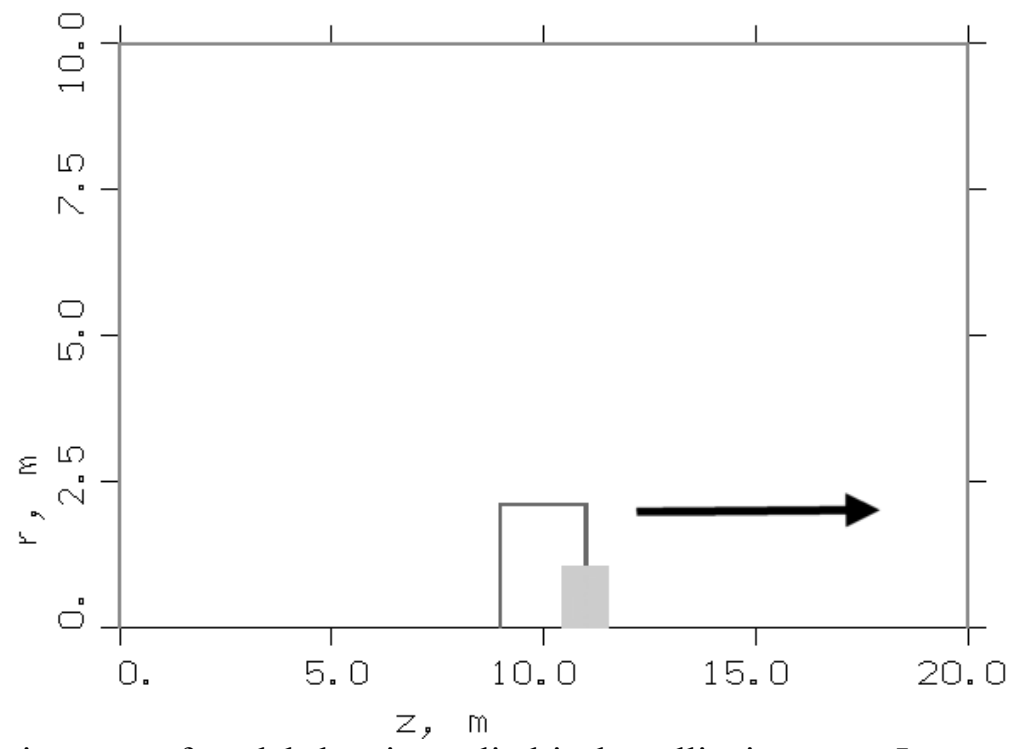

Figure 1 Geometric set up of model showing cylindrical satellite in centre. Ions are emitted from the right hand surface of the shaded region, with the arrow showing the direction of the ion beam.

The selfconsistent KARAT solution of Maxwell's equations and particle motion show that the spacecraft charges to potentials of $\mathrm{kV}$ in times of order $\mu \mathrm{s}$ (with a typical ion current of $\sim 10 \mathrm{~mA}$ ), causing back-streaming of the ion beam, Figure $2 \mathrm{a}$. This reduces the effectiveness of the ion thruster, by peturbing the potential well propelling the ions away from the spacecraft.
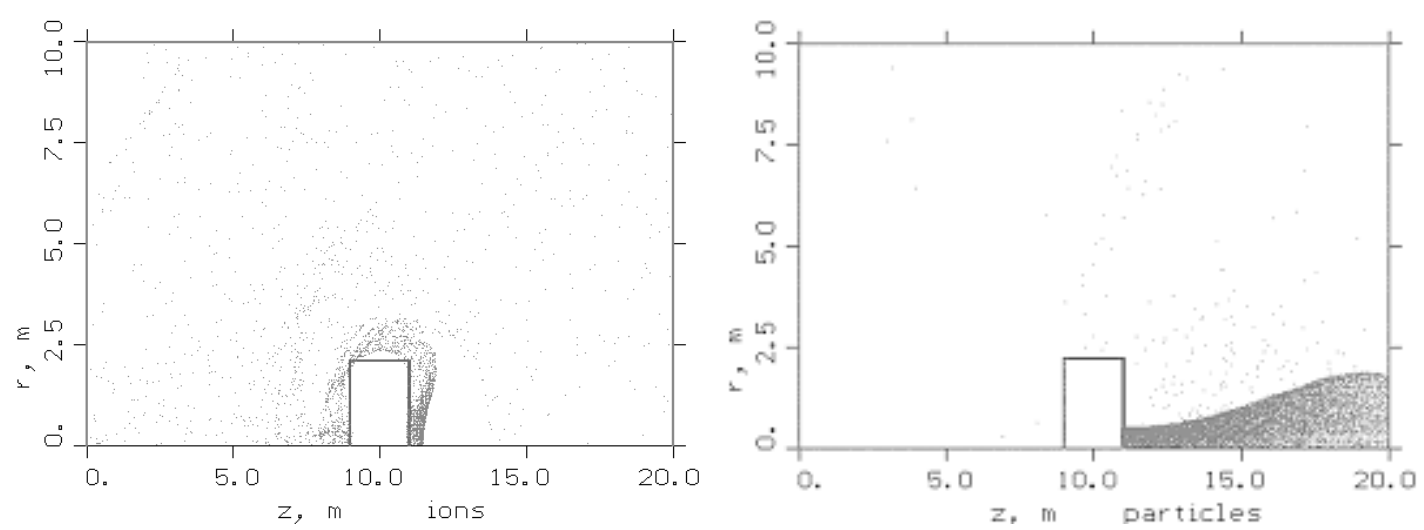

Figure 2 a) Equilibrium ion beam trajectory without a neutraliser. Ions are shown as dots. b)

Equilibrium trajectory of ion beam with neutraliser positioned at $r=2.5 \mathrm{~m}, z=10 \mathrm{~m}$. 
Inclusion of a neutraliser emitting a few $\mathrm{mA}$ restores the ion beam trajectory, Figure $2 \mathrm{~b}$. In this scale of model the field emitter neutraliser is simply defined as a surface emitting $100 \mathrm{eV}$ electrons. The RAL specification is to neutralise an ion current of $6 \mathrm{~mA}$ from four FEEP thrusters. KARAT runs have shown that only partial neutralisation occurs if the neutraliser current is less than the ion current. If the neutraliser current is equal to the ion current, neutralisation occurs in microseconds. Although the process can be accelerated if there is an excess of electrons, the RAL neutraliser is designed to emit $6 \mathrm{~mA}$, the same as the thruster.

The effect of neutraliser location in this model geometry was found to be minimal. However a key assumption is the homogeneity of conductivity of objects represented in the model. For an application with varying conductivity, such as a real satellite, it is recommended that the neutraliser and thruster are as close together as possible. This simple example demonstrates the necessity for a neutraliser.

\section{$4 \quad$ Laboratory Simulations}

\subsection{Electron emission technology}

Field emission of electrons from silicon nanotips is the core technology used in the neutraliser project. Space is a relatively new application for field emission, but RAL has already developed a miniaturised mass spectrometer using field emission for the ROSETTA mission [4]. RAL also has in-house construction capability at the Central Microstructures Facility, who fabricate the micromachined devices [5].

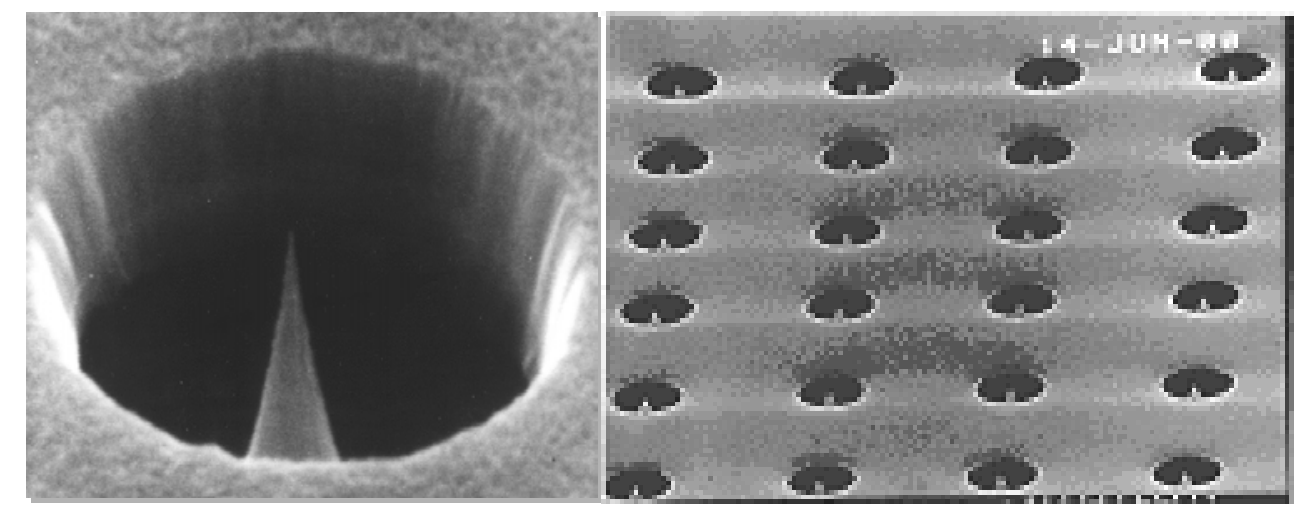

Figure 3 (a) Left- array of single crystal silicon field emitters with integrated gates produced at RAL CMF. (b) Right - magnified SEM single emitter tip (tip radius $<10 \mathrm{~nm}$ ). The aperture shown is $2 \mu \mathrm{m}$ diameter

Electrons are emitted from the tips (Figure 3a) (held at ground potential) if a positive voltage of up to $200 \mathrm{~V}$ is applied to the gate structure (Figure 3a,b) to accelerate the electrons away from the emitting surface. In space, the electrons are attracted towards the positive charge of the ion thruster, but in laboratory tests there is no ion thruster so a Faraday cup at a positive potential of $\sim 300 \mathrm{~V}$ is used to collect the emitted electrons. Laboratory measurements take place in a cylindrical vacuum tank at a pressure of $10^{-6}$ torr. Electrons are emitted from a gated field emitter about $1 \mathrm{~cm}$ away from the Faraday cup. 


\subsection{Modelling the laboratory test environment}

The laboratory environment model is on a smaller scale than the satellite model discussed in Section 3. The gate is represented in KARAT by a fine positively charged grid close to the field emitters. Electrons are emitted with energy of $5 \mathrm{eV}$, (comparable to the work function of the material) from a small surface to simulate field emission. $100 \mathrm{~V}$ is applied to the gate to accelerate the electrons. The geometry of the cylindrical vacuum tank is readily established, and currents at the electrodes (i.e. gate and Faraday cup), and particle trajectories were monitored.

One experiment decreased the Faraday cup (collector) potential to zero to investigate the effects of running the neutraliser without the ion beam switched on, which is the proposed initial switch on operation mode of the final neutraliser. The emitted electrons moved to the gate in preference to the collector. The experiment was simulated in KARAT, and the modelled results concurred with experimental observations of the gate current increasing and collected current decreasing as the collector voltage decreased. In both model and experiment, no current reached the collector when it was at zero potential.

The confidence in the model gained from the general agreement between experiment and simulation allows KARAT to be used for more detailed study of electron motion. Modelled electron trajectories gave further physical insight. With a small collector potential, selfrepulsion very quickly prevents any more electrons from reaching the cathode, and an abrupt change of direction can be seen. At zero collector potential, all the electrons oscillate between the field emitter and gate.

\section{Space Environment Simulations}

The successful simulation of laboratory experiments gives credence to model runs based on the space environment, which are less easily tested. As the neutraliser lifetime may be affected by bombardment from ions and neutrals, it is important to simulate the effects of background particle concentrations. Although an electromagnetic model cannot simulate the physical effects of the neutrals on the delicate field emitter array, KARAT can be used to predict the location and concentration of the neutral particles. KARAT can also simulate effects of the background plasma on neutralisation efficiency by modelling interactions between the charged particles emitted by the thruster and neutraliser.

The two most probable orbits for future neutraliser applications are low earth orbit (LEO) and geosynchronous orbit (GEO). LEO is within the thermosphere, or ionosphere, where atomic oxygen is the dominant constituent [6]. GEO is the height at which the satellite orbits in phase with the earth and makes one rotation every 24 hours.

\begin{tabular}{|l|l|l|l|}
\hline Orbit & $\begin{array}{l}\text { Distance from } \\
\text { Earth } \mathbf{( k m )}\end{array}$ & $\begin{array}{l}\text { Background plasma } \\
\left.\text { concentration } \mathbf{( c m}^{-3}\right)\end{array}$ & $\begin{array}{l}\text { Background } \\
\left.\text { concentration } \mathbf{( c m}^{-3}\right)\end{array}$ \\
\hline Low Earth & $<1000$ & $10^{4}-10^{5}$ & $10^{8}$ \\
\hline Geosynchronous & 35800 & 1 & $0.1 ?$ \\
\hline
\end{tabular}

Table 1 Background particle concentrations in possible neutraliser orbits (from [6] and [7])

The first use of the neutraliser on the ESA SMART-2 satellite, scheduled for launch in 2005, is likely to be in GEO or GEO-like orbit. However the worst case for potential degradation of the neutraliser is LEO, from the effects of atomic oxygen bombardment. As future missions may also be in LEO, there is a need to quantify effects of background particle concentrations in different orbits. It is possible to use the parameters in Table 1 as input plasma and neutral concentrations in KARAT. Inelastic processes are modelled using a Monte-Carlo method, with one species of neutral particles included; cross-sections of important ionisation processes are also parameterized [3]. 


\subsection{Low Earth Orbit}

In the KARAT simulation the number of particle species was restricted to four, so it is not possible to directly simulate LEO where five species of neutral and charged particles are under consideration. The problem is therefore broken down into two cases a) background and satellite-emitted charged particle interactions b) anthropogenic charged particles and ambient neutral particles.

\section{a) Charged particle interactions}

An orbit height of $500 \mathrm{~km}$ in the middle of the LEO range was chosen; the background plasma consists principally of $10^{4}-10^{5} \mathrm{~cm}^{-3}$ each of electrons and UV-ionised oxygen (with a smaller fraction of nitrogen ions, not included in this simulation) [8]. In KARAT, positive ions were set to have a relative atomic mass of $1^{1}$. Neutralisation is minimally affected by the background charge-neutral plasma. However, as the charged particle density is sensitive to the time of day and solar cycle [8] further simulations are required. Advection of plasma is also significant in the upper ionosphere [6] and should be included. In preliminary KARAT runs without a plasma source term, the background plasma self-neutralised in $20 \mu \mathrm{s}$, indicating that ionisation and advection processes are not yet adequately represented.

\section{b) Neutral particle interactions}

Neutralisation efficiency is unperturbed by neutral $\mathrm{O}$ atoms, though the process is tens of microseconds slower than in a true vacuum. Some ionisation occurs in the model: after $12.5 \mu \mathrm{s}$, a pocket of positive charge developed outside the usual beam path with $10^{8}$ particles $\mathrm{cm}^{-1}$ compared to $10^{10} \mathrm{~cm}^{-1}$ in the beam path. These are likely to have been formed by a wellknown ionisation process between an electron and neutral species [9], producing a positive ion and two electrons.

\subsection{Geosynchronous orbit}

GEO was simulated with charged particle concentrations as in Table 1; neutral particles were not included as concentrations are negligible and poorly defined. The background electrons migrated towards the ion beam but their peak concentration, located in the densest part of the ion beam was $10^{6} \mathrm{~cm}^{-1}$ compared to $10^{8} \mathrm{~cm}^{-1}$ for the neutraliser electrons at the same position. Therefore background electrons contribute a maximum of $1 \%$ to the ion beam neutralisation.

\section{Conclusions}

KARAT can effectively reproduce spacecraft charging with a FEEP thruster. Use of a $100 \mathrm{eV}$ electron beam as a simulated neutraliser discharges the spacecraft's negative charge; neutraliser position is unimportant for a uniformly conducting surface. The neutraliser should match the ion current of $6 \mathrm{~mA}$. Laboratory results were successfully modelled on a smallerscale simulation including a positive grid to accelerate electrons away from a low-energy electron source. In the absence of the ion thruster, electrons are expected to return to the acceleration electrode (gate) - a necessary result, as the neutraliser will be switched on before the ion thruster on the satellite. Neutralisers should therefore be built to withstand the full electron emission current returning to the gate.

\footnotetext{
${ }^{1}$ The model particles move a factor of $\sqrt{M}$ faster than a particle with a relative atomic mass of $M$ due to their increased mobility. This speeds up the model runs, and is physically equivalent to using more massive particles in longer model runs.
} 
Effects of two different orbit conditions on the neutraliser's electrical behaviour have been considered. Ambient neutral particles in LEO did not affect neutraliser operation, but recombination hindered plasma modelling. A source term needs to be included to assess the effect of background charged particles. GEO background plasma contributed a maximum of $1 \%$ to neutralisation. These preliminary model runs suggest a neutraliser is necessary and will function effectively in both its prospective orbits.

\section{Further Work}

It has been suggested that in the lower LEO range the plasma concentrations are so high that a neutraliser is not needed. Since background electrons do contribute slightly to neutralisation in GEO, there is a need to extend this study and assess the implications for neutraliser deployment. To do this, particle source and advection terms need to be defined to improve these plasma simulations.

Further ion thruster physics is required for a more realistic simulation. Thruster development work suggests that charged and neutral $\mathrm{Ce}$ atoms and clusters are emitted from the ion thruster, and reactions involving these particles are likely to have the most significant implications for the neutraliser. One way to improve the simulation of ion generation from the currently assumed degenerate, uniform ion beam would be to set up a detailed geometry of the ion thruster. Output of this model such as ion energy and spatial distribution will then be used to input to larger scale simulations.

\section{Acknowledgement}

This project is funded by the European Space Agency.

\section{References}

[1] Khodataev Y K, Bingham R, Tarakanov V P and Tsytovich V N, Plasma Phys Rep 22, 11, 932$942(1996)$

[2] Eloy M, Azambuja R, Mendonca J T and Bingham R Phys of Plasmas, 8, 3, 1084 (2001)

[3] Tarakanov V P, User's Manual for Code KARAT v 8.05, Moscow, Russia (2002)

[4] Kent B J, Huq E, Dominey J N and Morse A D, The use of microfabricated field emitter arrays in a high precision mass spectrometer for the Rosetta mission, Presented at the 3rd Round Table on Micro/Nano Technologies for Space, ESTEC, The Netherlands (2000)

[5] Huq S E, Kent B J, Stevens R, Lawes R A, Xu N S and She J C, J. Vac. Sci. Tech B. 19, 3, 988$991(2001)$

[6] Tribble A C, The space environment: implications for spacecraft design, Princeton University Press, Princeton, New Jersey (1995)

[7] Wynn-Williams G, The fullness of space, Cambridge University Press, Cambridge (1992)

[8] MacGorman D R and Rust W D The electrical nature of storms, Oxford University Press, New York (1998)

[9] Smirnov B M, Physics of ionized gases, Wiley, New York (2001) 